\title{
THERMAL MOTION IN SODIUM NITRITE: THE TEMPERATURE DEPENDENCE OF DEBYE- WALLER FACTORS
}

\author{
Y. Kawamura*, N. Nitta, H. Kasano and H. Mashiyama \\ Department of Physics, Faculty of Science, Yamaguchi University, \\ Yamaguchi 753-8512, Japan
}

(Received 2 September 2001)

In order to understand the relation between the transition mechanism and the thermal vibration, the temperature dependence of the Debye-Waller factor is investigated in $\mathrm{NaNO}_{2}$ by single-crystal $\mathrm{X}$-ray diffractometry. The crystal parameters are refined by least squares calculations both in paraelectric and ferroelectric phases. The maximum entropy method is also employed to estimate the spontaneous polarization. The Debye-Waller factor displays a minor anomaly at the order-disorder transition point. The result is compared with $\mathrm{K}_{2} \mathrm{SeO}_{4}$, a typical displacive crystal.

Keywords: Debye-Waller factor; order-disorder/displacive type; sodium nitrite; structure analysis; least squares calculation; maximum entropy method

\section{INTRODUCTION}

Two types of transition mechanism have been recognized in dielectric crystals; the order-disorder type and the displacive type. ${ }^{[1]}$ From a view point of crystal structure analysis, a split-atom method is successfully applied to the former, while an atom has a definite position with the anisotropic thermal parameters in the latter.

The thermal motion is represented by Debye-Waller (D-W) factors in the structural analysis, where the temperature factor is represented by

$$
\begin{aligned}
T=\exp \{ & -2 \pi^{2}\left[U_{11}\left(h a^{*}\right)^{2}+U_{22}\left(k b^{*}\right)^{2}+U_{33}\left(l c^{*}\right)^{2}\right. \\
+ & \left.\left.2 U_{12} h a^{*} k b^{*}+2 U_{13} h a^{*} l c^{*}+2 U_{23} k b^{*} l c^{*}\right]\right\} .
\end{aligned}
$$

\footnotetext{
${ }^{*}$ Corresponding author, e-mail: b4342@ sty.cc.yamaguchi-u.ac.jp
} 
The square root of $U_{\mathrm{jj}}$ represents the effective amplitude of the thermal vibration along the $j$-axis of the crystal.

It has been sometimes stated that the D-W factor behaves differently in order-disorder and in displacive type crystals. ${ }^{[2,3]}$ Figure 1 shows a schematic behavior of the D-W factor. In the former, the D-W factor (marked by closed circles $U_{2}$ ) drops steeply at the structural transition. In the latter, the D-W factor (open circles $U_{3}$ ) would have a cusp-type anomaly at the transition.

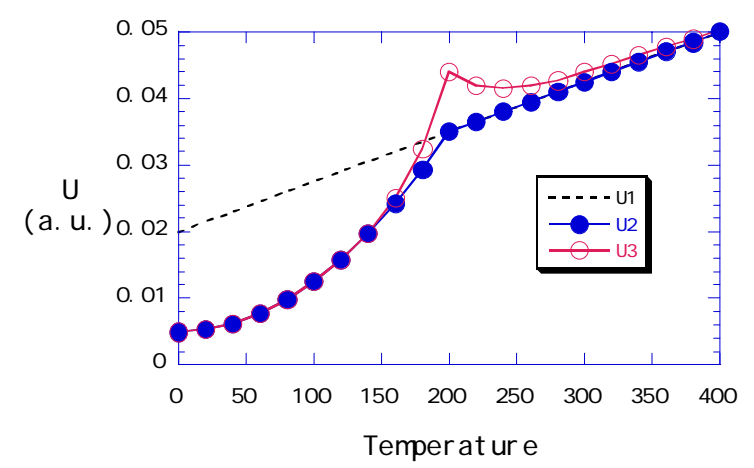

Figure 1. Schematic behavior of the Debye-Waller factor. The Transition is presumed at $T=200$.

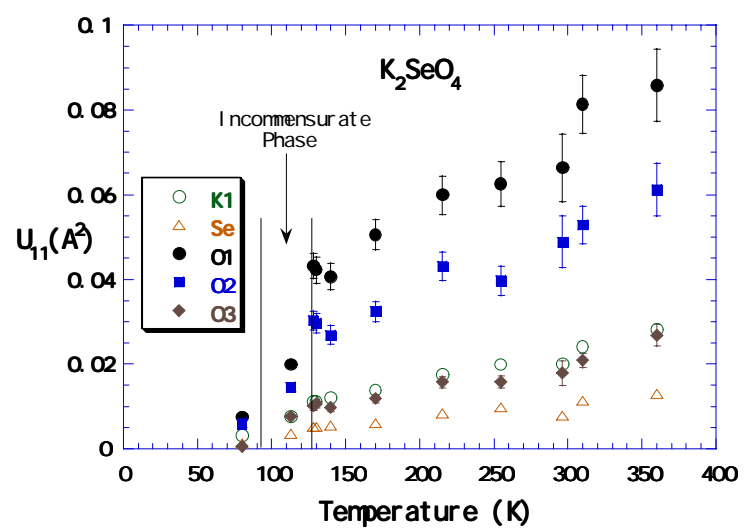

Figure 2. The Debye-Waller factor $U_{11}$ of $\mathrm{K}_{2} \mathrm{SeO}_{4}$. The data points at $115 \mathrm{~K}$ and $80 \mathrm{~K}$ are after Yamada et al.(1984). ${ }^{[5,6]}$ The esd's are shown by bars.

Although the conjecture sounds plausible, we have found that the D$\mathrm{W}$ factors of $\mathrm{K}_{2} \mathrm{SeO}_{4}$, a typical incommensurate crystal, which has a soft phonon mode, decrease monotonically with decreasing temperature, and behave just as closed circles of Fig. 1. If we choose the space group of $\mathrm{K}_{2} \mathrm{SeO}_{4}$ as Pmcn, then the dominant displacement is along the 
$a$-axis. The temperature dependence of the $\mathrm{D}-\mathrm{W}$ factor $U_{11}$ is shown in Fig. $2{ }^{[4]}$, although the structural transition at $128 \mathrm{~K}$ is the displacive type.

Since our result is in contradiction to the previous conjecture, we investigate the temperature dependence of $\mathrm{D}-\mathrm{W}$ factor in $\mathrm{NaNO}_{2}$, a typical incommensurate crystal that performs the order-disorder type transition. ${ }^{[7]}$ At room temperature the space group is $\operatorname{Im} 2 m(Z=2)$. The spontaneous polarization vanishes at $437 \mathrm{~K}$, above which the incommensurate phase exists in a narrow temperature range (less than $2 \mathrm{~K}){ }^{[8]}$ In the high-temperature paraelectric phase, the $\mathrm{NO}_{2}$ molecule takes the up-down orientated state with equal probability to ascertain the mirror symmetry.

\section{EXPERIMENT AND RESULT}

Small pieces of $\mathrm{NaNO}_{2}$ were cut from the transparent part of single crystals. Each specimen was sealed in a glass capillary to avoid moisture and was mounted on a four-circle X-ray diffractometer. Atomic parameters including D-W factors were refined by least squares calculations. A split-atom method was employed to represent the updown orientated state in the paraelectric phase for all constituent atoms.

Figure 3 shows the temperature dependence of D-W factors for three atoms. The previously reported parameters by several authors are also plotted in the figure; $30 \mathrm{~K},{ }^{[9]} 120 \mathrm{~K},{ }^{[10]} 295 \mathrm{~K},{ }^{[1]} 338 \mathrm{~K},{ }^{[12]} 378 \mathrm{~K},{ }^{[12]}$ $418 \mathrm{~K},{ }^{[12]} 443 \mathrm{~K},{ }^{[13]} 458 \mathrm{~K}^{[14]}$ and $498 \mathrm{~K}^{[14]}$. Other 26 temperature points are obtained for 4 samples by our analysis. The temperature region of the incommensurate phase is excluded. Our results are in coincidence with the previous ones essentially. The large amplitude motion of the $\mathrm{NO}_{2}$ molecule normal to the molecular plane, $U_{11}$, suggests that the updown motion of the dipole moment takes place through the rotation of the $\mathrm{NO}_{2}$ molecule about an axis parallel to the $c$-axis. ${ }^{[13]}$

The scatters at room temperature indicate a sample dependence. In the paraelectric phase, the correlation between the split positions and the thermal parameters induces some scatters in $U_{\mathrm{j} j}$. However, the temperature dependence can be expressed as the following; (1) $U_{11}$ and $U_{33}$ vary with temperature continuously through the transition. (2) $U_{22}$ behaves as $U_{2}$ of Fig. 1; a small kink can be recognized at the transition. 

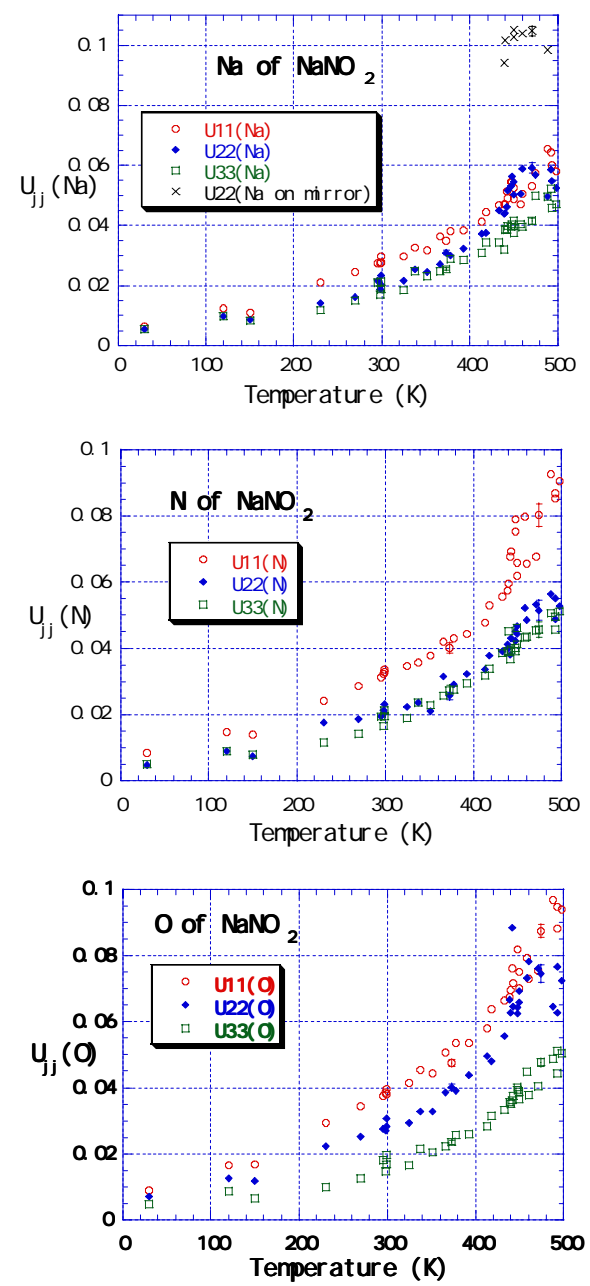

Figure 3. The temperature dependence of the Debye-Waller factors of $\mathrm{NaNO}_{2}$.

In the paraelectric phase, the Fourier synthesis shows double peaks for $\mathrm{N}$ and elongated peaks for $\mathrm{O}$ and $\mathrm{Na}$, which indicates disordered nature. If the atomic position of $\mathrm{Na}$ is fixed on the mirror plane, then $U_{22}$ becomes about twice of the split-atom as shown by crosses in the top of Fig. 3.

\section{DISCUSSIONS}

As mentioned in the introduction, the plausible arguments claimed that $U_{2}$ and $U_{3}$ of Fig. 1 corresponded to order-disorder and displacive types, 
respectively. Our analysis for $\mathrm{NaNO}_{2}$ shows that $U_{22}$, order-disorder in character, is the type $U_{2}$ and that $U_{11}$ and $U_{33}$ are almost continuous through the transition. The displacive crystal, $\mathrm{K}_{2} \mathrm{SeO}_{4}$, also showed the behavior of the type $U_{2}$.

Some years ago, a theoretical calculation was performed to demonstrate the temperature dependence of the $\mathrm{D}-\mathrm{W}$ factor; the types of $U_{3}$ in Fig. 1 is realized in order-disorder type transitions, while $U_{2}$ in displacive type transition. ${ }^{[15]}$ Our results for displacive type crystal coincide with the theoretical prediction. If the local potential of an atom would not change at the order-disorder transition, the D-W factor would decrease almost continuously around the transition. If the splitatom method was not applied, the D-W factor was the type of $U_{3}$.

In addition to this conclusion, we discuss two points; the order parameter and the spontaneous polarization. Just below the transition, the constituent atoms are not fully ordered; there remains the fraction of split atoms in the ferroelectric phase. So we determined the occupation probability of split atoms as a fitting parameter in the least squares calculations. The result coincided with those obtained by Gohda et al. ${ }^{[12]}$ If we ignored the fraction of split atoms, then $U_{22}$ of $\mathrm{N}$ became more than twice larger than that of Fig. 3 just below the transition temperature.

Finally we calculate the spontaneous polarization in ferroelectric phase. By means of least squares calculations, we determine the center of charge density of each atom. If we assigned the net charges for $\mathrm{Na}, \mathrm{N}$ and $\mathrm{O}$ as $+1,+3$ and -2 , then the calculated polarization was $78 \mu \mathrm{C} / \mathrm{cm}^{2}$. But this is much larger than $12 \mu \mathrm{C} / \mathrm{cm}^{2}$ estimated from the
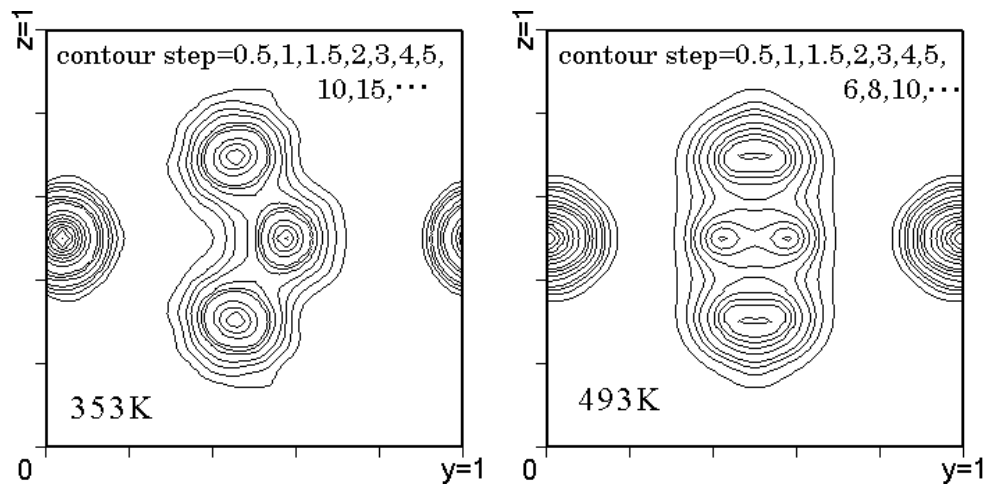

Figure 4. Electron density $(\mathrm{x}=1 / 2)$ of $\mathrm{NaNO}_{2}$ in ferroelectric phase $(353 \mathrm{~K})$ and in paraelectric phase (493K). 
direct measurement of the polarization. ${ }^{[16]}$ Gohda et al. have recently discussed this problem by using multi-pole type electron density function and they have got $7.8 \mu \mathrm{C} / \mathrm{cm}^{2} .^{[9]}$ This method assumed additional fitting parameters in the least squares calculations. We adopt another method; the maximum entropy method is superior, because no a priory assumption has been made on charge distribution. [17] The electron charge is determined by maximum entropy method at $353 \mathrm{~K}$, as given in Fig. 4. The net charge is estimated 1.08, 0.16 and 0.62 for $\mathrm{Na}, \mathrm{N}$ and $\mathrm{O}$, respectively. If the positions of nuclei are those of obtained by the usual least squares calculations, then the polarization is $18.1 \mu \mathrm{C} / \mathrm{cm}^{2}$, much closer to experimental value of the polarization.

Since the bond length $\mathrm{N}-\mathrm{O}$ is $1.251(2) \mathrm{A}$ at $353 \mathrm{~K}$, the empirical method of the bond valence sum ${ }^{[18]}$ predicts the bond valence to be 1.35(4). If this charge is transferred from $\mathrm{O}^{2-}$ to $\mathrm{N}^{3+}$, then the net charge of $\mathrm{O}$ becomes $-0.65(4)$ in agreement with our estimation.

\section{References}

[1] M. E. Lines and A. M. Glass, Principles and Applications of Ferroelectrics and Related Materials (Clarendon, Oxford, 1977).

[2] K. Itoh, H. Matsunaga and E. Nakamura, Phys. Status Solidi b97, 289 (1980).

[3] K. Itoh, J. Phys. Soc. Jpn. 53, 2049 (1984).

[4] N. Nitta, Master's Thesis (Yamaguchi Univ., 2001, unpublished).

[5] N. Yamada and T. Ikeda, J. Phys. Soc. Jpn. 53, 2555 (1984).

[6] N. Yamada, Y. Ono and T. Ikeda, J. Phys. Soc. Jpn. 53, 2565 (1984).

[7] S. Sawada, S. Nomura, S. Fujii and I. Yoshida, Phys. Rev. Letters 1, 320, (1958).

[8] S. Tanisaki, J. Phys. Soc. Jpn. 16, 579 (1961).

[9] T. Gohda, M. Ichikawa, T. Gustafsson and I. Olovsson, Acta Crystallogr. B 56, $11(2000)$.

[10] M. Okuda, S. Ohba, Y. Saito, T. Ito and I. Shibuya, Acta Crystallogr. B 46, 343 (1990).

[11] T. Gohda, M. Ichikawa, T. Gustafsson and I. Olovsson, J. Korean Phys. Soc. 32, S189 (1998).

[12] T. Gohda, M. Ichikawa, T. Gustafsson and I. Olovsson, Phys. Rev. B63, 14101 (2000).

[13] K. Komatsu, K. Itoh and E. Nakamura, J. Phys. Soc. Jpn. 57, 2836 (1988).

[14] M. I. Kay, J. A. Gonzalo and R. Maglic, Ferroelectrics 9, 179 (1975).

[15] K. Fujii and T. Matsubara, Prog. Theor. Phys. 78, 177 (1987).

[16] K. Hamano, J. Phys. Soc. Jpn. 35, 157 (1973).

[17] S. Kumazawa,Y. Kubota, M. Takata and M. Sakata, J. Appl. Cryst. 26, 453 (1993)

[18] I. D. Brown and D. Altermatt, Acta Cryst. B41, 241 (1985). 